UCRHEP-T437

August 2007

\title{
Observing the Dark Scalar Doublet and its Impact on the Standard-Model Higgs Boson at Colliders
}

\author{
Qing-Hong Cad* and Ernest Mad \\ Department of Physics and Astronomy, \\ University of California, Riverside, California 92521, USA \\ G. Rajasekarant \\ Institute of Mathematical Sciences, \\ Chennai (Madras) 600113, India
}

\begin{abstract}
If the Standard Model (SM) of particle interactions is extended to include a second scalar doublet $\left[H^{+},\left(H^{0}+i A^{0}\right) / \sqrt{2}\right]$, which is odd under an unbroken $Z_{2}$ discrete symmetry, it may be called the dark scalar doublet, because its lightest neutral member, say $H^{0}$, is one posssible component for the dark matter of the Universe. We discuss the general phenomenology of the four particles of this doublet, without assuming that $H^{0}$ is the dominant source of dark matter. We also consider the impact of this dark scalar doublet on the phenomenology of the SM Higgs boson $h$.
\end{abstract}

\footnotetext{
*Electronic address: qcao@ucr.edu

${ }^{\dagger}$ Electronic address: ernest.ma@ucr.edu

${ }^{\ddagger}$ Electronic address: graj@imsc.res.in
} 


\section{INTRODUCTION}

The canonical Standard Model (SM) of quarks and leptons requires only one Higgs doublet $\left(\phi^{+}, \phi^{0}\right)$, such that $\phi^{0}$ acquires a nonzero vacuum expectation value $\left\langle\phi^{0}\right\rangle=v=174 \mathrm{GeV}$, from which all particles (except neutrinos) obtain mass. Suppose a second scalar doublet $\left[H^{+},\left(H^{0}+i A^{0}\right) / \sqrt{2}\right]$ is added [1], which is odd under an unbroken $Z_{2}$ discrete symmetry, then there are at least two interesting outcomes.

(I) This scalar doublet also has a conserved additive quantum number, so that $\left(H^{0}+\right.$ $\left.i A^{0}\right) / \sqrt{2}$ is a mass eigenstate, i.e. $m_{H^{0}}=m_{A^{0}}$. Such is in fact the case of supersymmetry, where this is nothing but the complex conjugate of a scalar lepton doublet. More generally, it may belong to a new class of particles [2, 3] with a separately conserved additive quantum number unrelated to lepton number.

(II) There is no additional symmetry beyond the postulated $Z_{2}$, in which case $m_{H^{0}} \neq m_{A^{0}}$. As pointed out [1] already in 1977, either $H^{0}$ or $A^{0}$ must then be stable. This idea has been revived recently, first in the context of radiative neutrino masses [4], then as a means of understanding precision electroweak data [5]. In any case, this specific potential new source of dark matter of the Universe [6] is gaining more attention [7, 8, 9, 10, 11, 12]. It will also have an important bearing on interpreting forthcoming data from the LHC (Large Hadron Collider) at CERN.

In this paper we will analyze in detail the two-scalar-doublet structure of scenario (II), which we call DSDM (Dark Scalar Doublet Model). Whereas there are specific constraints from identifying $H^{0}$ as the sole source of dark matter [5, 9], we will take the viewpoint that there may also be other sources of dark matter such as that coming from supersymmetry. For example, the first such explicit model with two (and possibly three) different dark-matter particles has already been proposed [13]. Thus our analysis will allow a wider range of parameter values, with the aim of extracting the possible signals of this dark scalar doublet at the LHC. This doublet has been called the "inert Higgs doublet" [5, 9], but it is neither inert (since it has gauge and scalar interactions) nor a contributor to the Higgs mechanism (since it has no vacuum expectation value), hence we prefer to call it the dark scalar doublet.

Consider the scalar potential of $\Phi_{1}=\left(\phi^{+}, \phi^{0}\right)$ and $\Phi_{2}=\left[H^{+},\left(H^{0}+i A^{0}\right) / \sqrt{2}\right]$, with $\Phi_{1}$ even and $\Phi_{2}$ odd under an exactly conserved $Z_{2}$ :

$$
V=\mu_{1}^{2} \Phi_{1}^{\dagger} \Phi_{1}+\mu_{2}^{2} \Phi_{2}^{\dagger} \Phi_{2}+\frac{1}{2} \lambda_{1}\left(\Phi_{1}^{\dagger} \Phi_{1}\right)^{2}+\frac{1}{2} \lambda_{2}\left(\Phi_{2}^{\dagger} \Phi_{2}\right)^{2}+\lambda_{3}\left(\Phi_{1}^{\dagger} \Phi_{1}\right)\left(\Phi_{2}^{\dagger} \Phi_{2}\right)
$$




$$
+\lambda_{4}\left(\Phi_{1}^{\dagger} \Phi_{2}\right)\left(\Phi_{2}^{\dagger} \Phi_{1}\right)+\frac{1}{2} \lambda_{5}\left(\Phi_{1}^{\dagger} \Phi_{2}\right)^{2}+\frac{1}{2} \lambda_{5}^{*}\left(\Phi_{2}^{\dagger} \Phi_{1}\right)^{2}
$$

All the above parameters are necessarily real except $\lambda_{5}$, but the exact $Z_{2}$ symmetry (which forbids the bilinear term $\Phi_{1}^{\dagger} \Phi_{2}$ allows us to rotate the relative phase between $\Phi_{1}$ and $\Phi_{2}$ to make $\lambda_{5}$ real as well. As the standard $S U(2) \times U(1)$ gauge symmetry is spontaneously broken by $\left\langle\phi^{0}\right\rangle=v=174 \mathrm{GeV}$, the masses of the resulting scalar particles are given by [4]

$$
\begin{aligned}
m^{2}(h) & =2 \lambda_{1} v^{2}, \\
m^{2}\left(H^{ \pm}\right) & =\mu_{2}^{2}+\lambda_{3} v^{2}, \\
m^{2}\left(H^{0}\right) & =\mu_{2}^{2}+\left(\lambda_{3}+\lambda_{4}+\lambda_{5}\right) v^{2} \\
m^{2}\left(A^{0}\right) & =\mu_{2}^{2}+\left(\lambda_{3}+\lambda_{4}-\lambda_{5}\right) v^{2} .
\end{aligned}
$$

The lone Higgs boson of the Standard Model is of course $h$, whereas $H^{ \pm}, H^{0}$, and $A^{0}$ are the components of the dark scalar doublet which interact with $h$ and among themselves as follows:

$$
\begin{aligned}
V_{i n t} & =\frac{1}{2} \lambda_{2}\left[H^{+} H^{-}+\frac{1}{2}\left(H^{0}\right)^{2}+\frac{1}{2}\left(A^{0}\right)^{2}\right]^{2}+\lambda_{3}\left(v h+\frac{1}{2} h^{2}\right)\left[H^{+} H^{-}+\frac{1}{2}\left(H^{0}\right)^{2}+\frac{1}{2}\left(A^{0}\right)^{2}\right] \\
& +\frac{1}{2}\left(\lambda_{4}+\lambda_{5}\right)\left(v h+\frac{1}{2} h^{2}\right)\left(H^{0}\right)^{2}+\frac{1}{2}\left(\lambda_{4}-\lambda_{5}\right)\left(v h+\frac{1}{2} h^{2}\right)\left(A^{0}\right)^{2} .
\end{aligned}
$$

Being electroweak doublets, they also have gauge interactions, but they do not interact directly with quarks or leptons.

Assuming the mass hierarchy $m_{H^{ \pm}}>m_{A^{0}}>m_{H^{0}}$, the stable $H^{0}$ then appears as missing energy in the decays of $A^{0}$ and $H^{ \pm}$. Since there is no term linear in $A^{0}$ or $H^{0}$ in Eq. (6), the decay of $A^{0}$ must occur through the gauge interaction

$$
\frac{g}{2 \cos \theta_{W}} Z_{\mu}\left(H^{0} \partial^{\mu} A^{0}-A^{0} \partial^{\mu} H^{0}\right) .
$$

Hence the dominant decay of $A^{0}$ is into $H^{0} f \bar{f}$, where $f=$ lepton or quark, and $H^{0}$ is missing energy.

As for $H^{ \pm}$, its gauge interactions with $H^{0}$ and $A^{0}$ are given by

$$
\frac{i g}{2} W_{\mu}^{-}\left(H^{0} \partial^{\mu} H^{+}-H^{+} \partial^{\mu} H^{0}\right)+\frac{g}{2} W_{\mu}^{-}\left(A^{0} \partial^{\mu} H^{+}-H^{+} \partial^{\mu} A^{0}\right)+\text { h.c. }
$$

Hence the dominant decays of $H^{ \pm}$are into $W^{ \pm} H^{0}$ and $W^{ \pm} A^{0}$, with the subsequent decay of $A^{0}$ into $Z^{0} H^{0}$ in the latter.

The only other trilinear gauge interactions of the dark scalars are those of $H^{ \pm}$with $Z$ and $\gamma$. There are of course also the quadrilinear terms required by gauge invariance. 


\section{LEP CONSTRAINTS}

\section{A. Z-boson decay}

If $m_{H^{0}}+m_{A^{0}}<m_{Z}$, then $Z$ can decay into $H^{0} A^{0}$ with the subsequent decay of $A^{0}$ to $H^{0} f \bar{f}$ where $f$ is a quark or lepton. This would appear as a rare $Z$ decay event with $f \bar{f}+$ missing energy in the final state. The high precision LEP-I experiments have accumulated a sample of about $1.7 \times 10^{7}$ on-shell $Z$ boson decays via the $q \bar{q}$ and $\ell^{+} \ell^{-}$channels [14]. ALEPH preformed a partial analysis of an integrated $79 \mathrm{pb}^{-1}$ to search for the rare decay $Z \rightarrow f \bar{f}+\nu \bar{\nu}$ and found no event above the SM prediction [15]. To estimate how the whole LEP-I data sample can constrain the DSDM, we calculate the SM partial decay width of $Z \rightarrow f \bar{f}+\nu \bar{\nu}(f=q, \ell)$ and obtain its decay branching ratio

$$
\begin{aligned}
\mathrm{BR}(Z \rightarrow f \bar{f}+\nu \bar{\nu}) & =\frac{\left(4.269_{q \bar{q} \nu \bar{\nu}}+0.779_{\ell^{+} \ell^{-} \nu \bar{\nu}}\right) \times 10^{-7} \mathrm{GeV}}{2.494_{\text {total }} \mathrm{GeV}} \\
& \simeq 2.02 \times 10^{-7} .
\end{aligned}
$$

We thus expect about $\left(1.7 \times 10^{7}\right) \times\left(2.0 \times 10^{-7}\right) \simeq 3.4$ background events from the SM. Assuming that only 4 events show up as $f \bar{f}+$ missing energy from the whole LEP-I data sample, we deduce according to Possion statistics that only about 5 signal events are allowed at $95 \%$ C.L. in the $f \bar{f}+H^{0} H^{0}$ channel, i.e.

$$
\frac{\Gamma\left(Z \rightarrow f \bar{f}+H^{0} H^{0}\right)}{\Gamma_{0}} \leq \frac{5}{1.7 \times 10^{7} \times 0.8} \simeq 3.7 \times 10^{-7},
$$

where the SM branching ratio $B R(Z \rightarrow f \bar{f}) \simeq 0.8$ (for $f=q, \ell$ ) has been used. This implies that the phase space for $Z \rightarrow H^{0} A^{0}$ is very small (with a mass gap of a few MeV), so for all practical purposes, the bound $m_{H^{0}}+m_{A^{0}}>m_{Z}$ should be respected [11]. The resulting lower limits on $m_{H^{0}}$ for various $\Delta m_{A^{0} H^{0}} \equiv m_{A^{0}}-m_{H^{0}}$ are shown in Table \.

TABLE I: Lower limits on $m_{H^{0}}$ from the LEP $Z$-decay constraint for various $\Delta m_{A^{0} H^{0}}$.

\begin{tabular}{cccccc}
\hline$\Delta m_{A^{0} H^{0}}(\mathrm{GeV})$ & 5 & 10 & 15 & 20 & 30 \\
\hline \hline$m_{H^{0}}(\mathrm{GeV})$ & 43 & 41 & 38 & 36 & 31 \\
\hline
\end{tabular}

Since $A^{0} \rightarrow H^{0}+\nu \bar{\nu}$ is also possible, this process may also contribute to the invisible width of the $Z$ if $m_{H^{0}}+m_{A^{0}}<m_{Z}$. However, the corresponding experimental uncertainty allows for a mass gap of a few $\mathrm{GeV}$, which is not competitive with the constraint discussed above. 


\section{B. Neutralino search at LEP}

While there is no existing experimental search that can be directly translated into a search for the dark scalars, the search for charginos and neutralinos is very close as they may exhibit the same collider signatures. The OPAL Collaboration [16] has performed a search for neutralino pair production via the process of $e^{+} e^{-} \rightarrow \widetilde{\chi}_{2}^{0} \widetilde{\chi}_{1}^{0}$ in the hadronic decay mode $\tilde{\chi}_{2}^{0} \rightarrow \widetilde{\chi}_{1}^{0} Z^{*} \rightarrow \widetilde{\chi}_{1}^{0} q q$ where $\tilde{\chi}_{1,2}^{0}$ denotes the lightest (next lightest) neutralino, respectively. No significant excess above the SM background leads to an upper limit on the cross section of $\widetilde{\chi}_{2}^{0} \widetilde{\chi}_{1}^{0}$ associated production: $\sigma_{\widetilde{\chi}_{1}^{0} \widetilde{\chi}_{2}^{0}} \sim 0.1 \mathrm{pb}-0.5 \mathrm{pb}$ for $m_{\widetilde{\chi}_{1}^{0}} \sim 50 \mathrm{GeV}$ and $m_{\tilde{\chi}_{2}^{0}} \sim 60-80 \mathrm{GeV}$ (see the right figure of Fig. 10 in Ref. [16]). The L3 Collaboration [17] also performed a similar search at $\sqrt{s}=189 \mathrm{GeV}$, which gives the upper limit on the cross section as $\sigma_{\widetilde{\chi}_{1}^{0} \widetilde{\chi}_{2}^{0}} \sim 0.1 \mathrm{pb}-2 \mathrm{pb}$ for $m_{\widetilde{\chi}_{1}^{0}} \sim 50 \mathrm{GeV}$ and $m_{\tilde{\chi}_{2}^{0}} \sim 60-80 \mathrm{GeV}$ (see Fig. 3a in Ref. [17]).

The translation of the above limits on the cross section from the neutralino to the dark scalar is not straightforward even though they share the same topologies. The kinematics and spin correlations of $\widetilde{\chi}_{1}^{0} \widetilde{\chi}_{2}^{0}$ and $A^{0} H^{0}$ pair productions are totally different. For example, the scalar pair has to be in the $p$-wave as it is produced via a virtual $Z$-boson, therefore, each scalar has large transverse momentum [18]. Furthermore, there is no spin correlation between the two scalars. Those differences lead to different acceptances of the kinematics cuts used by the OPAL and L3 Collaborations to extract the signal from the background. One thus cannot simply apply the OPAL and L3 constraints to the DSDM. In order to get the realistic limits, one has to go through the whole analysis using the correct kinematics and spin correlations. For a rough estimation, we now simply compare the prediction of the DSDM to the OPAL and L3 limits. The production cross section of $e^{+} e^{-} \rightarrow A^{0} H^{0}$ in the DSDM at $\sqrt{s}=208 \mathrm{GeV}$ is about $0.28 \mathrm{pb}-0.20 \mathrm{pb}$ for $m_{H^{0}}=50 \mathrm{GeV}$ and $m_{A^{0}}=60 \mathrm{GeV}-80 \mathrm{GeV}$. It is reasonably consistent with both OPAL and L3 constraints. Hence, we will use these masses in the following monte carlo study.

\section{Higgs direct search}

If the $Z_{2}$-odd scalars are lighter than the SM Higgs boson, they may have a great impact on the direct search of the latter because $h \rightarrow H^{0} H^{0}$ and $h \rightarrow A^{0} A^{0}$ may become the 

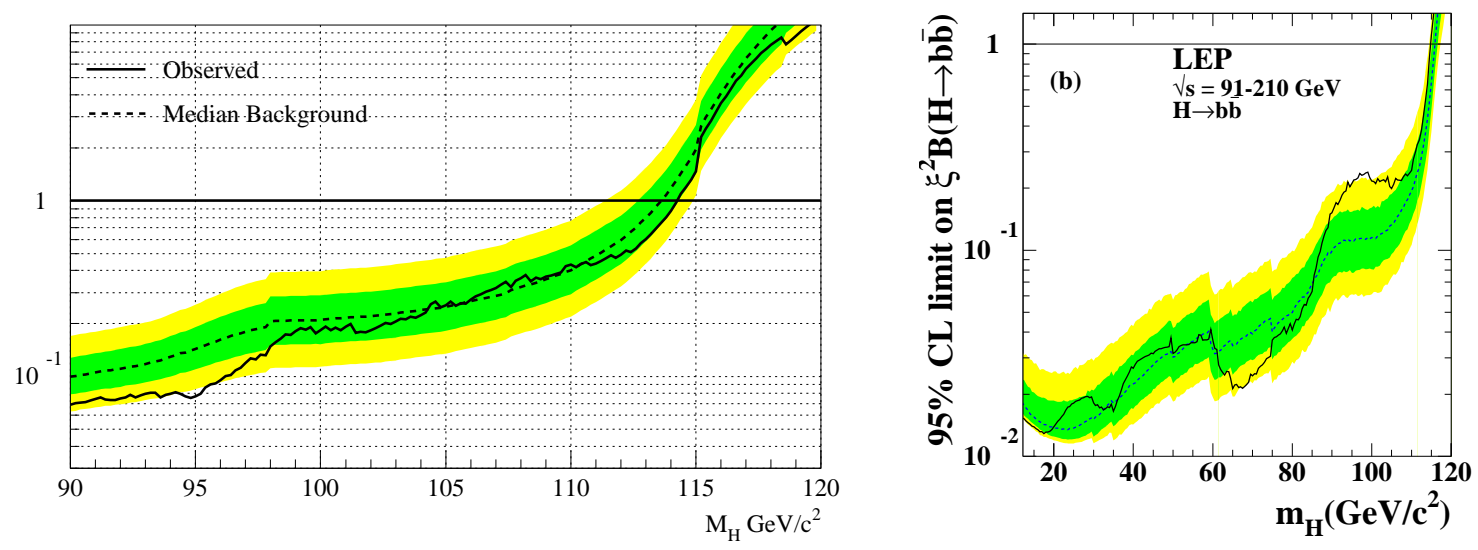

FIG. 1: (left) The 95\% C.L. upper limit on $B R(h \rightarrow$ invisible $)$, adapted from [19]; (right) The 95\% C.L. upper limit on $B R(h \rightarrow b \bar{b})$, adapted from [20]. The label H in these figures refers to the SM Higgs boson $h$, and $\xi^{2}$ in the right figure is defined as $\xi^{2} \equiv\left(g_{H Z Z} / g_{H Z Z}^{S M}\right)^{2}$ (see Ref. [20] for details).

dominant decay channels. In particular, the $h \rightarrow H^{0} H^{0}$ channel is invisible. LEP II has performed a direct search of the SM Higgs boson in its invisible decay mode but found no event, thus obtaining an upper bound on this decay branching ratio, shown on the left in Fig. 1. Below we will use this information to find the allowed parameter space of the DSDM. As for the $h \rightarrow A^{0} A^{0}$ decay mode, it may give rise to four charged leptons plus missing energy, resulting from $A^{0} \rightarrow H^{0} Z^{*} \rightarrow H^{0} \ell^{+} \ell^{-}$. At LEP the SM Higgs boson is produced in association with an on-shell $Z$-boson, i.e. $e^{+} e^{-} \rightarrow Z h$, which decays into two charged leptons or two jets. Thus, if the SM Higgs boson decays into two $A^{0}$ bosons, there could be a collider signature of six charged leptons plus missing energy, or four charged leptons plus two jets plus missing energy. A new scan over the accumulated data sample at LEP to search for such a signature may prove to be interesting.

LEP II searched the SM Higgs boson mainly via the process $e^{+} e^{-} \rightarrow Z\left(\rightarrow \ell^{+} \ell^{-}\right) h(\rightarrow b \bar{b})$ as the decay $h \rightarrow b \bar{b}$ dominates for a light Higgs boson. The negative search at LEP II gives rise to the upper bound on the decay branching ratio of $h \rightarrow b \bar{b}$, shown in the right in Fig. 1. and sets a lower limit of the SM Higgs boson mass of $m_{h}>114.4 \mathrm{GeV}$. However, this limit can be relaxed in the DSDM because the invisible decay of the Higgs boson may be 
dominant so that the decay branching ratio into $b \bar{b}$ is highly suppressed ${ }^{1}$.

The LEP II bounds on $h \rightarrow$ invisible and $h \rightarrow b \bar{b}$ compete with each other. The former requires smaller invisible decay branching ratio while the latter requires larger. Consider now the possibility of the DSDM satisfying both limits.

The partial decay width of $h_{S M} \rightarrow b \bar{b}$ is given by

$$
\Gamma\left(h_{S M} \rightarrow b \bar{b}\right)=\frac{N_{C} m_{b}^{2} m_{h}}{8 \pi v^{2}}\left(1-\frac{4 m_{b}^{2}}{m_{h}^{2}}\right)^{3 / 2},
$$

while the partial decay width of $h_{S M} \rightarrow S S$ ( $S$ denoting a member of the dark scalar doublet) is given by

$$
\Gamma(h \rightarrow S S)=\delta_{S} \frac{v^{2}}{16 \pi m_{h}} \lambda_{S}^{2} \sqrt{1-\frac{4 m_{S}^{2}}{m_{h}^{2}}},
$$

where $\delta_{H^{0} / A^{0}}=1$ and $\delta_{H^{+}}=2$, and the coupling $\lambda_{S}$ is given by

$$
\lambda_{H^{0}}=\lambda_{3}+\lambda_{4}+\lambda_{5}, \quad \lambda_{A^{0}}=\lambda_{3}+\lambda_{4}-\lambda_{5}, \quad \lambda_{H^{+}}=\lambda_{3} .
$$

From Eqs. (3+51), the above couplings can be further simplified as

$$
\lambda_{H^{0}}=\frac{m_{H^{0}}^{2}-\mu_{2}^{2}}{v^{2}}, \quad \lambda_{A^{0}}=\frac{m_{A^{0}}^{2}-\mu_{2}^{2}}{v^{2}}, \quad \lambda_{H^{+}}=\frac{m_{H^{+}}^{2}-\mu_{2}^{2}}{v^{2}} .
$$

Thus, the partial decay width of the SM Higgs boson, given in Eq. 9, only depends on two parameters, $m_{S}$ and $\mu_{2}$, for a given $m_{h}$. We note that $\mu_{2}$ is not a free parameter because of the requirement of vacuum stability, i.e.

$$
\lambda_{1,2}>0 ; \quad \lambda_{3}, \lambda_{3}+\lambda_{4}-\left|\lambda_{5}\right|>-\sqrt{\lambda_{1} \lambda_{2}}
$$

Hence we obtain the following bound on $\mu_{2}$,

$$
\mu_{2}^{2}<m_{H^{0}}^{2}+\sqrt{\frac{\lambda_{2}}{2}} m_{h} v,
$$

which reveals the fact that the self-interaction of the dark scalar doublet, i.e. $\lambda_{2}$, has a nontrivial impact on the SM sector. As to be shown later, the stronger the dark sector interacts, the larger is the allowed parameter space of the DSDM for the SM Higgs boson.

Assuming the charged Higgs boson mass is much larger than $m_{h}$ and $m_{A^{0}}-m_{H^{0}}=10 \mathrm{GeV}$, we now scan over the parameter space of the DSDM. In summary, the following conditions need to be satisfied in order to relax the LEP limit $m_{h}>114.4 \mathrm{GeV}$ :

\footnotetext{
${ }^{1}$ Similar studies in various models have been carried out in Refs. 21, 22, 23, 24, 25].
} 
- LEP II bound, $B R_{\max }(i n v)$, on $h \rightarrow$ invisible

$$
B R(i n v) \equiv \frac{\Gamma\left(h_{S M} \rightarrow H^{0} H^{0}\right)}{\Gamma\left(h_{S M} \rightarrow b \bar{b}\right)+\Gamma\left(h_{S M} \rightarrow H^{0} H^{0}\right)+\Gamma\left(h_{S M} \rightarrow A^{0} A^{0}\right)}<B R_{\max }(i n v) .
$$

- LEP II bound, $B R_{\max }(b \bar{b})$, on $h \rightarrow b \bar{b}$

$$
B R(b \bar{b}) \equiv \frac{\Gamma\left(h_{S M} \rightarrow b \bar{b}\right)}{\Gamma\left(h_{S M} \rightarrow b \bar{b}\right)+\Gamma\left(h_{S M} \rightarrow H^{0} H^{0}\right)+\Gamma\left(h_{S M} \rightarrow A^{0} A^{0}\right)}<B R_{\max }(b \bar{b}) .
$$

- Z-decay constraint

$$
m_{Z}<m_{H^{0}}+m_{A^{0}} .
$$

- Vacuum stability bound given in Eq. 13,

We note that the vacuum stability bound is highly correlated with the bound on the decay of $h \rightarrow b \bar{b}$. It can be understood as follows. Due to the $Z$-pole constraint $m_{H^{0}}$ should be larger than $41 \mathrm{GeV}$ for $\Delta m_{A^{0} H^{0}}=10 \mathrm{GeV}$, therefore, the SM-like Higgs boson has to be heavier than $82 \mathrm{GeV}$ in order to open the $h \rightarrow H^{0} H^{0}$ mode. Bearing in mind the fact that $B R_{\max }(b \bar{b})<0.1$ for a light SM-like Higgs boson, e.g. $m_{h} \sim 100 \mathrm{GeV}$, the LEP II bound on $h \rightarrow b \bar{b}$ now becomes

$$
\frac{3 m_{b}^{2} m_{h}}{8 \pi v^{2}} \beta_{b}^{3 / 2}<B R_{\max }(b \bar{b}) \frac{v^{2}}{16 \pi m_{h}}\left(\frac{m_{H^{0}}-\mu_{2}^{2}}{v^{2}}\right)^{2} \beta_{H^{0}}
$$

where $\beta_{b}=\sqrt{1-4 m_{b}^{2} / m_{h}^{2}}$ and $\beta_{H^{0}}=\sqrt{1-4 m_{H^{0}}^{2} / m_{h}^{2}}$. As $\beta_{H} \sim 0$ for a light Higgs boson, $\mu_{2}$ has to be very large, even larger than $m_{H^{0}}$, in order to satisfy the above inequality. On the other hand, $\mu_{2}$ is also bounded from above due to vacuum stability. It thus leads to an interesting connection between the vacuum stability bound and the LEP II bound on $h \rightarrow b \bar{b}$.

The allowed region of $\lambda_{2}$, which satisfies the constraints on both $B R_{\max }(i n v)$ and $B R_{\max }(b \bar{b})$, is shown in Fig. 2(a). For the sake of illustration, we plot the allowed parameter space of $\left(m_{H^{0}}, \mu_{2}\right)$ for $m_{h}=109 \mathrm{GeV}$ and $112 \mathrm{GeV}$ in Fig. 2(b) and (c). In the DSDM the lower limits of $m_{h}$ are shown in Table $\amalg$ for various $\Delta m_{A^{0} H^{0}}$. 

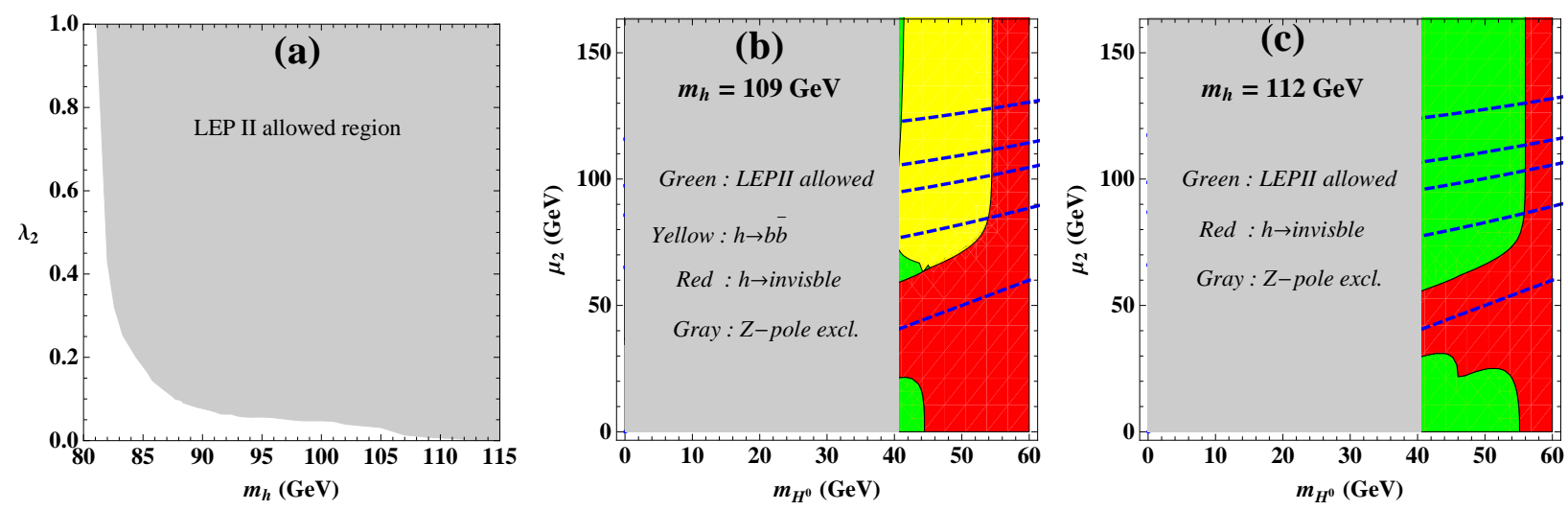

FIG. 2: (a) Allowed parameter space (gray region) of $\left(m_{h}, \lambda_{2}\right)$; (b)(c) allowed parameter space of $\left(m_{H^{0}}, \mu_{2}\right)$ after imposing the LEP II constraints on the decay branching ratio of $h \rightarrow$ invisible and $h \rightarrow b \bar{b}$. The region above the dashed line is excluded by the vacuum stability requirement. From top to bottom: $\lambda_{2}=1.0,0.5,0.3,0.1$ and $\lambda_{2} \rightarrow 0$.

TABLE II: Lower limits on the mass of the SM Higgs boson derived from LEP II Higgs boson search for various $\Delta m_{A^{0} H^{0}}$.

\begin{tabular}{cccccc}
\hline$\Delta m_{A^{0} H^{0}}(\mathrm{GeV})$ & 5 & 10 & 15 & 20 & 30 \\
\hline$m_{h}(\mathrm{GeV})$ & 105 & 106 & 109 & 110 & 110 \\
\hline
\end{tabular}

\section{IMPACT ON THE SM HIGGS SEARCH AT COLLIDERS}

At the LHC, the SM Higgs boson is mainly produced through gluon-gluon fusion induced by a heavy (top) quark loop. Once produced, it will decay into a fermion pair or vector boson pair. The strategy of searching for the Higgs boson depends on how it decays and how large the decay branching ratio is. We present selected decay branching ratios of the SM Higgs boson as a function of $m_{h}$ in Fig. 3(a), as well as the total decay width $\left(\Gamma_{t o t}\right)$ in units of $\mathrm{GeV}$. If the SM Higgs boson is lighter than $130 \mathrm{GeV}$, it decays mainly into a bottom quark pair $(b \bar{b})$. Unfortunately, it is very difficult to search for the Higgs boson in this mode due to the extremely large Quantum Chromodynamics (QCD) background at the LHC. However, the $h \rightarrow \gamma \gamma$ mode can be used to detect a Higgs boson with the mass below $150 \mathrm{GeV}$ [26, 27] even though the decay branching ratio of this mode is quite small, $\sim O\left(10^{-3}\right)$. If the Higgs boson mass $\left(m_{h}\right)$ is in the region of $130 \mathrm{GeV}$ to $2 m_{Z}$, the $h \rightarrow Z Z^{*}$ mode is very useful because of its clean collider signature of four isolated charged leptons. 

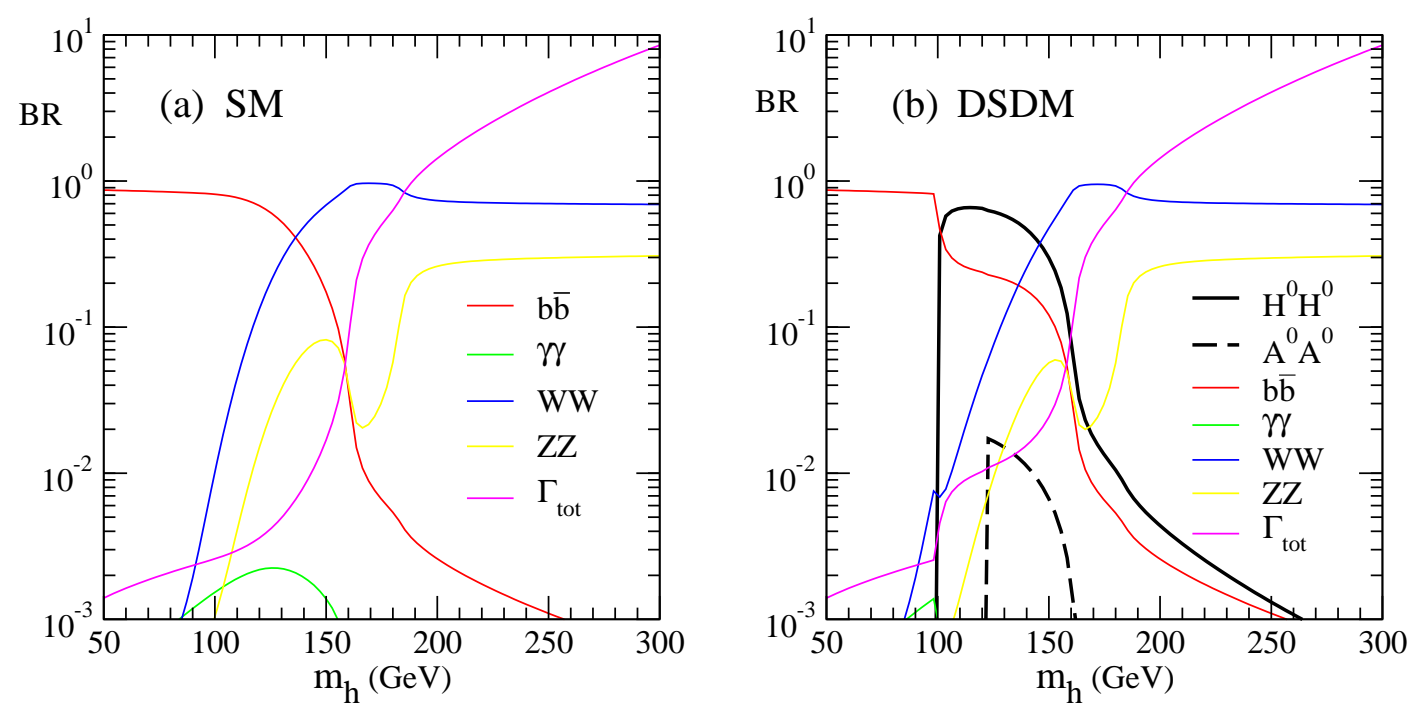

FIG. 3: (a) Selected Higgs boson decay branching ratios as a function of $m_{h}$ in the SM; (b) selected SM Higgs boson decaying ratios as a function of $m_{h}$ in the DSDM. Here we have chosen $m_{H^{0}}=50 \mathrm{GeV}, \Delta m_{A^{0} H^{0}}=10 \mathrm{GeV}, m_{H^{ \pm}}=170 \mathrm{GeV}$ and $\mu_{2}=20 \mathrm{GeV}$. The vertical axis is units of $\mathrm{GeV}$ for the total decay width.

The $h \rightarrow W W^{(*)}$ mode is also important in this mass region because of its large decay branching ratio [28]. if $m_{h}>2 m_{Z}$, the decay mode $h \rightarrow Z Z \rightarrow \ell^{+} \ell^{-} \ell^{\prime+} \ell^{\prime-}$ is considered as the "gold-plated" mode which is the most reliable way to detect the Higgs boson up to $m_{h} \sim 600 \mathrm{GeV}$ because the backgrounds are known rather precisely and the two on-shell $Z$ bosons could be reconstructed experimentally. For $m_{h}>600 \mathrm{GeV}$, one can detect the $h \rightarrow Z Z \rightarrow \ell^{+} \ell^{-} \nu \bar{\nu}$ decay channel in which the signal appears as a Jacobian peak in the missing transverse energy spectrum [29]. In this section we show a few representive cases to illustrate how the new dark scalars affect the Higgs search at the LHC. Our choice of masses for all the particles is not necessarily constrained by precision electroweak data [5, 30]. The numerical calculation is carried out with the help of the HDECAY program [31], after the implementation of the new decay channels in the DSDM.

The new decay channels of the SM Higgs boson will increase its decay width but decrease the decay branching ratios of the usual decay modes, such as $b \bar{b}, W W, Z Z$ and $\gamma \gamma$, etc. In Fig. 3(b) we present the relevant decay branching ratios of the SM Higgs boson as a function of its mass. Clearly, the usual decay modes are highly suppressed once the $H^{0} H^{0}$ and $A^{0} A^{0}$ decay modes open. The invisible mode $\left(h \rightarrow H^{0} H^{0}\right)$ dominates in the light and intermediate 

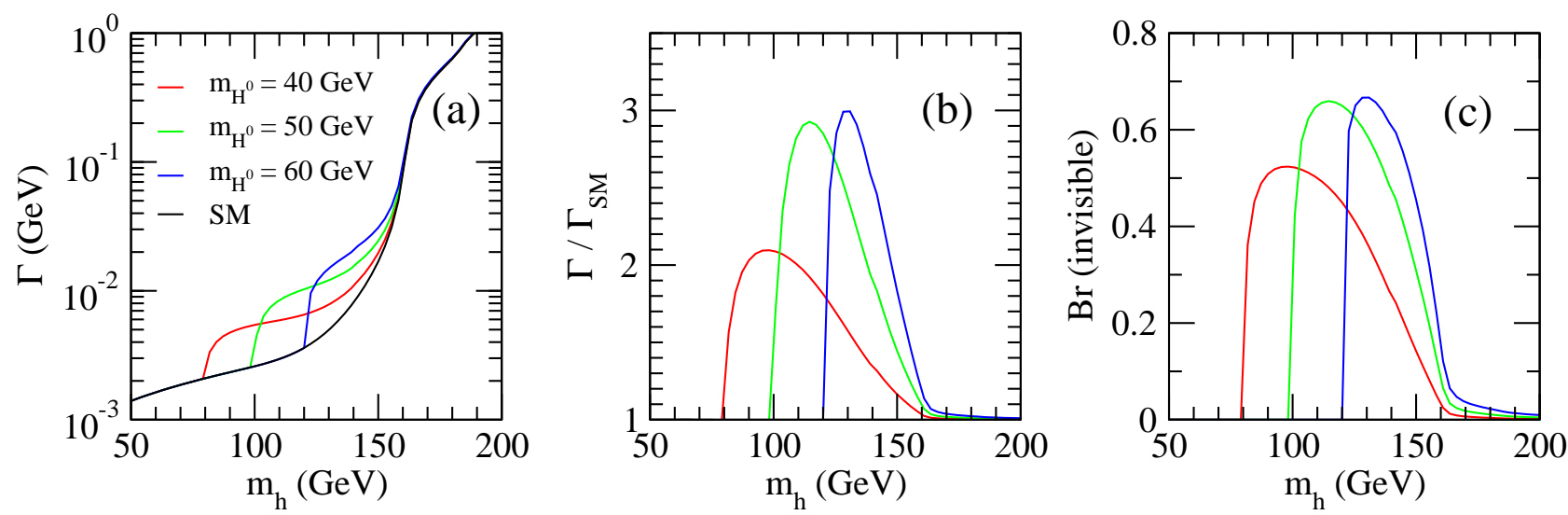

FIG. 4: (a) Total decay width of the SM Higgs boson as a function of $m_{h}$; (b) ratio of the total decay width of the SM Higgs boson in the DSDM and Higgs boson in the SM; (c) decay branching ratio of the invisible decay mode of the SM Higgs boson in the DSDM. For comparison, we choose $m_{H^{0}}=40(50,60) \mathrm{GeV}, \Delta m_{A^{0} H^{0}}=10 \mathrm{GeV}, m_{H^{ \pm}}=170 \mathrm{GeV}$, and $\mu_{2}=20 \mathrm{GeV}$.

mass region of $m_{h}$, i.e. $100<m_{h}<160 \mathrm{GeV}$. Due to the $10 \mathrm{GeV}$ mass gap between $H^{0}$ and $A^{0}$, the contribution of the $H^{0} H^{0}$ mode is much larger than that of the $A^{0} A^{0}$ mode, as shown by the black solid and dashed curves in Fig. 3(b). It implies that the SM Higgs boson decay depends mainly on $m_{H^{0}}$ but not $m_{A^{0}}$. We also note that the $\gamma \gamma$ mode is suppressed so much that it will be very challenging to use this mode to detect the SM Higgs boson in the mass region of $m_{h} \sim 100-150 \mathrm{GeV}$.

To examine the dependence of the SM Higgs decay upon $m_{H^{0}}$, we choose sample points of $m_{H^{0}}$ being $40(50,60) \mathrm{GeV}$ and present the corresponding Higgs boson total decay width, the ratio of total decay width in the DSDM to the one in the $\mathrm{SM}\left(\Gamma / \Gamma_{S M}\right)$ and the decay branching ratio of the invisible decay mode $(B r$ (invisible)) in Fig. 4(a) (b) and (c), respectively. Clearly, the total decay width increases by a big amount once the new decay channel opens. For example, as shown in Fig. 4(b), the new invisible decay mode can enlarge the total width by a factor of three for $m_{H^{0}}=60 \mathrm{GeV}$ and $m_{h}=130 \mathrm{GeV}$ and by a factor of two for $m_{H^{0}}=40 \mathrm{GeV}$ and $m_{h}=100 \mathrm{GeV}$. The decay branching ratio of the invisible mode is about $50-65 \%$ in the mass region $m_{h} \sim 100-150 \mathrm{GeV}$ for $m_{H^{0}} \sim 40-60 \mathrm{GeV}$. Needless to say, the $W W$ mode dominates over the invisible mode once the SM Higgs boson is heavier than $160 \mathrm{GeV}$, beyond which the SM Higgs boson in the DSDM is essentially the same as that of the SM. 

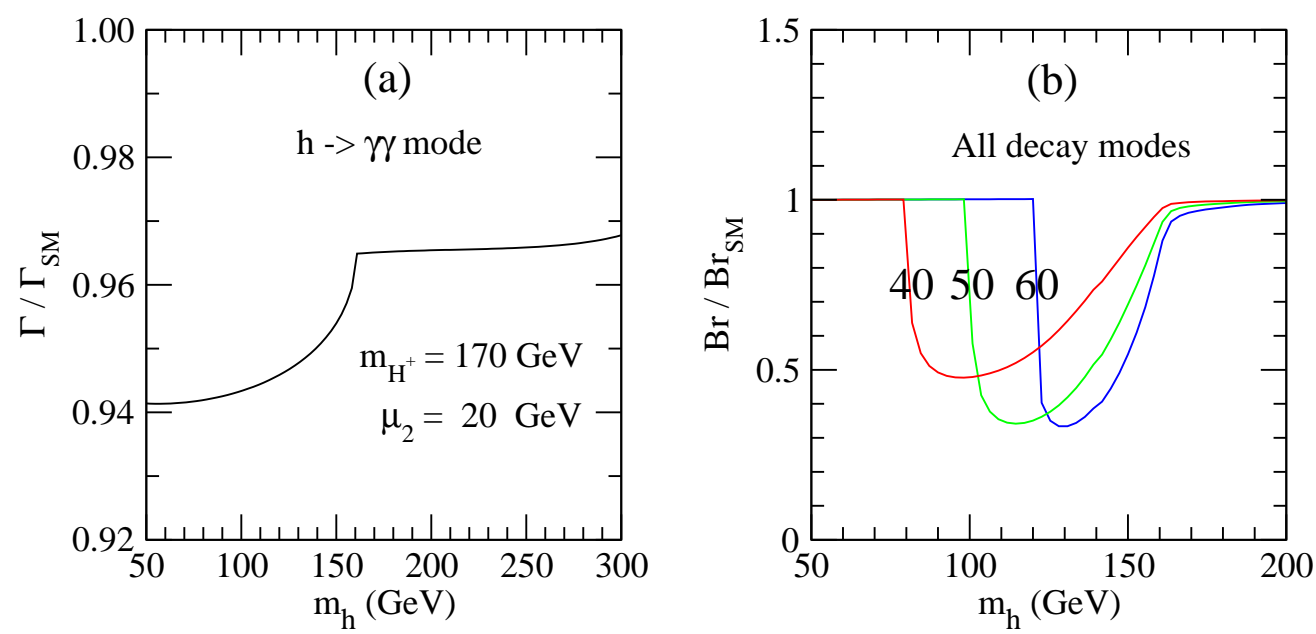

FIG. 5: (a) Ratio of the decay branching ratios of $h_{S M} \rightarrow \gamma \gamma$ in the DSDM and the SM; (b) suppression factor of the usual decay modes compared to the SM for $m_{H^{0}}=40,50,60 \mathrm{GeV}$. Here we have chosen $\Delta m_{A^{0} H^{0}}=10 \mathrm{GeV}$ and $\mu_{2}=20 \mathrm{GeV}$.

In the DSDM the decay mode of $h \rightarrow \gamma \gamma$ receives an additional contribution from the charged Higgs boson loop. The partial decay width of $h \rightarrow \gamma \gamma$ is given by [32, 33]

$$
\begin{aligned}
& \Gamma(h \rightarrow \gamma \gamma) \\
= & \frac{G_{\mu} \alpha^{2} m_{h}^{3}}{128 \sqrt{2} \pi^{3}}\left|\sum_{f} N_{C} Q_{f}^{2} g_{h f f} \mathcal{A}_{1 / 2}\left(\tau_{f}\right)+g_{h W W} \mathcal{A}_{1}\left(\tau_{W}\right)+\frac{m_{H^{ \pm}}^{2}-\mu_{2}^{2}}{\sqrt{2} m_{H^{ \pm}}^{2}} \mathcal{A}_{0}\left(\tau_{H^{ \pm}}\right)\right|^{2},
\end{aligned}
$$

where the amplitude $\mathcal{A}_{i}$ and coupling $g_{h f f / h W W}$ are given in Refs. [32, 33]. For the numerical calculation we also implemented the correction from the charged Higgs boson loop in the HDECAY program. The impact of the invisible decay on the other regular decay modes are shown in Fig. 5, We note that the loop correction of the charged Higgs boson slightly suppresses the partial decay width of $h \rightarrow \gamma \gamma$ by about 10\%, as shown in Fig. 5(a). Such a suppression makes it more difficult to search for the SM Higgs boson via the di-photon mode. Furthermore, as an overall effect, all the usual decay modes of the SM Higgs boson, e.g. $b \bar{b} / W W / Z Z / \gamma \gamma$, are highly suppressed once the new decay mode opens. In the intermediate mass regime, $130<m_{h}<160 \mathrm{GeV}$, the suppression factor can be larger than 0.5 which definitely makes the search of the SM Higgs boson more challenging.

On the other hand, one might search for the Higgs boson through its invisible decay mode. For example, the weak-boson fusion (WBF) process, $q \bar{q} \rightarrow q^{\prime} \bar{q}^{\prime} V V \rightarrow q^{\prime} \bar{q}^{\prime} h$ with the subsequential decay to undetectable particles, i.e. $h \rightarrow H^{0} H^{0} / A^{0} A^{0}$. The signal is thus 

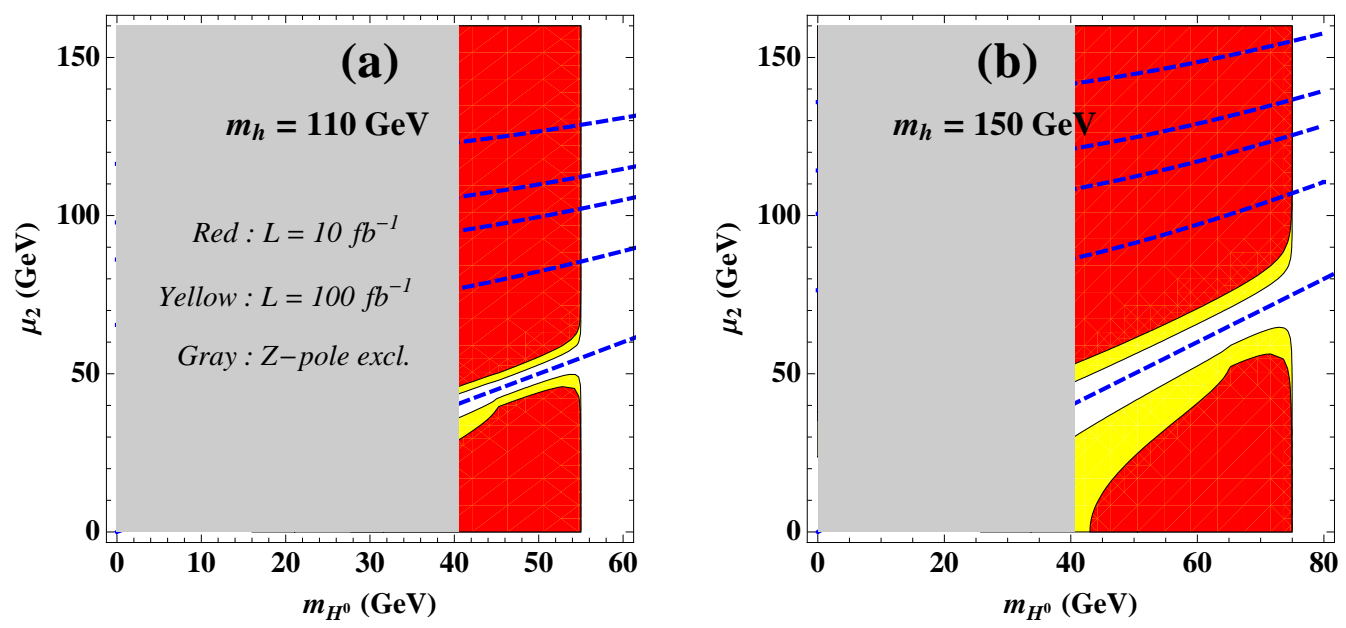

FIG. 6: Allowed parameter space of $\left(m_{H^{0}}, \mu_{2}\right)$ to reach the $5 \sigma$ discovery of the invisible Higgs decay via the WBF process: (a) $m_{h}=110 \mathrm{GeV}$, (b) $m_{h}=150 \mathrm{GeV}$. The region above the dashedlines is excluded by the vacuum stability requirement. From top to bottom: $\lambda_{2}=1.0,0.5,0.3,0.1$ and $\lambda_{2} \rightarrow 0$.

characterized by two quark jets, which typically stay in the foward and backward regions of the detector and are widely separated in pseudorapidity, and also by a large missing transverse momentum $\left(E_{T}\right)$, due to the Higgs invisible decay products. It has been shown in Ref. [34] that the WBF process can be used to detect the invisible decay Higgs boson at $5 \sigma$ level, when statistical errors only are considered, if the invisible decay branching ratio satisfies the following condition

$$
\frac{\sigma_{S} \times \operatorname{Br}(h \rightarrow \text { invisible }) \times \mathcal{L}}{\sqrt{\sigma_{B} \times \mathcal{L}}}>5,
$$

where $\mathcal{L}$ denotes the integrated luminosity and $\sigma_{S}\left(\sigma_{B}\right)$ the cross section of the signal (background) respectively. After imposing the kinematical cuts suggested in Ref. [34], $\sigma_{B}=167 \mathrm{fb}$ and $\sigma_{S}=99.4(99.7,94.3,89.2)$ fb for $m_{h}=110(120,130,150) \mathrm{GeV}$. From the above relation, we obtain the allowed parameter region of $\left(m_{H^{0}}, \mu_{2}\right)$ to reach the $5 \sigma$ discovery as shown in Fig. 6. Most of the parameter space can be covered for $\mathcal{L}=100 \mathrm{fb}^{-1}$, c.f. the yellow region.

Unfortunately, finding or constraining an invisibly decaying Higgs boson signal in $j j+\not_{T}$ event is essentially a counting experiment since a resonance in the invariant mass distribution of the Higgs decay products cannot be extracted. It also depends on two or more independent model parameters, thus one can only probe or constrain their combination. Next we are 
going to examine the dark scalar pair production at the LHC, which is complementary to the WBF process.

\section{COLLIDER PHENOMENOLOGY OF THE DARK SCALAR DOUBLET}

\section{A. Production of dark scalar pair at the LHC}

Since all new scalars carry a $Z_{2}$-odd quantum number, they cannot be produced singly at the collider. Furthermore, these new scalars only couple to the Higgs boson and electroweak gauge bosons of the Standard Model. Hence they should be produced via processes of the Drell-Yan type at the LHC as listed below:

$$
\begin{aligned}
& q \overline{q^{\prime}} \rightarrow W^{*} \rightarrow A^{0} H^{ \pm} \\
& q \overline{q^{\prime}} \rightarrow W^{*} \rightarrow H^{0} H^{ \pm} \\
& q \bar{q} \rightarrow Z^{*} \rightarrow A^{0} H^{0} \\
& q \bar{q} \rightarrow Z(\gamma) \rightarrow H^{+} H^{-} .
\end{aligned}
$$

In the DSDM, the vertex of $V-\phi-\phi^{\prime}$ is a pure gauge coupling, where $V$ denotes an SM gauge boson while $\phi\left(\phi^{\prime}\right)$ is a $Z_{2}$-odd scalar. Therefore, the total cross section of each production process depends only on the masses of the $Z_{2}$-odd scalars in the final state. In this work the matrix elements of both signal and background processes are calculated using MADGRAPH [35, 36] while the widths of the dark scalars are calculated in CALCHEP [37] with the model file generated by LANHEP [38]. Agreement of both programs has been checked at the level of dark scalar pair production. In Fig. 7 we present the contours of the cross sections (in fb units) of the $A^{0} H^{+}$and $A^{0} H^{0}$ associated production at the LHC in the plane of the scalars' masses. The red symbol "X" denotes one of our benchmark pionts, $\left[m_{H^{0}}, m_{A^{0}}, m_{H^{ \pm}}\right]=[50,60,175] \mathrm{GeV}$, used in the collider study. We note that the production cross section of the dark scalar pair at the LHC is sizable, e.g. at the benckmark point shown in Fig. 7 the cross section of the $A^{0} H^{0}$ pair production is about 3 pb while that

of the $A^{0} H^{ \pm}$pair production is about $200 \mathrm{fb}$. The latter is highly suppressed owing to the large mass of $H^{ \pm}$. Due to the same reason the cross section of the $H^{+} H^{-}$pair production is quite small in the relevant parameter space (around the benchmark piont); it is therefore not shown in this study. 


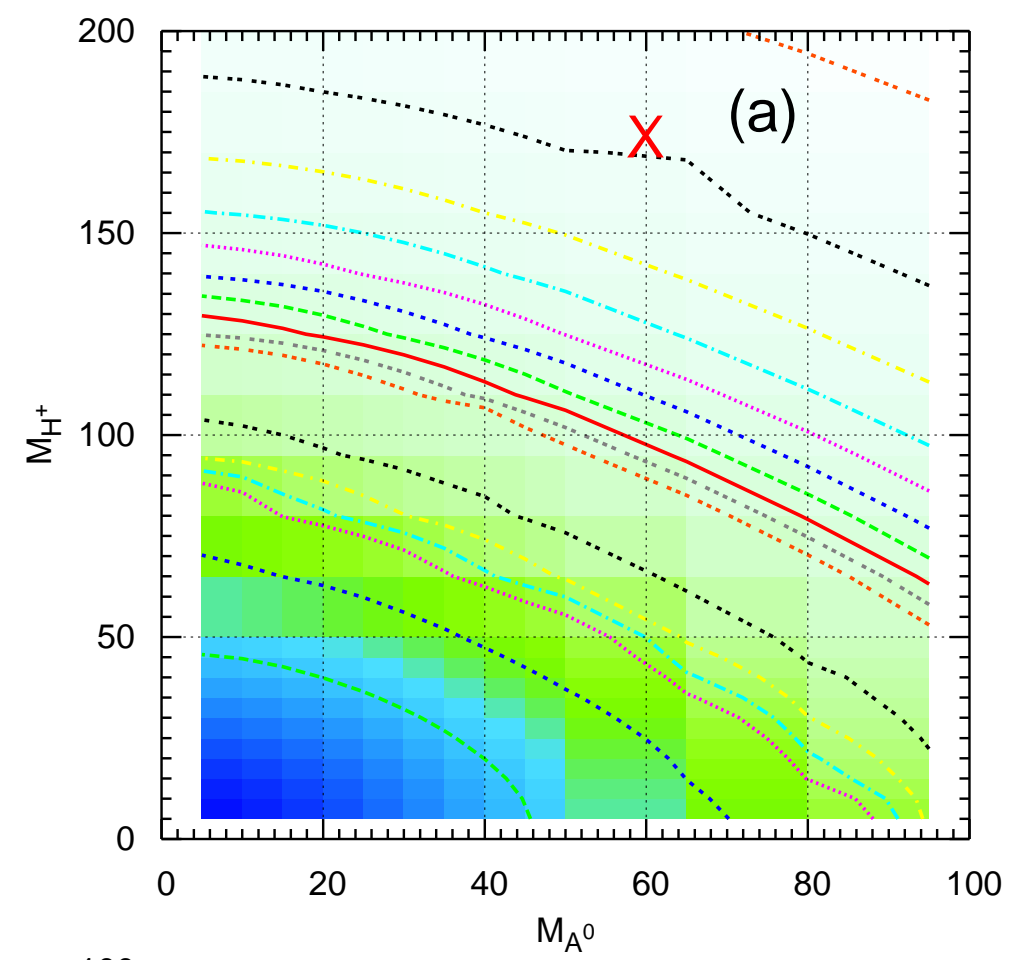

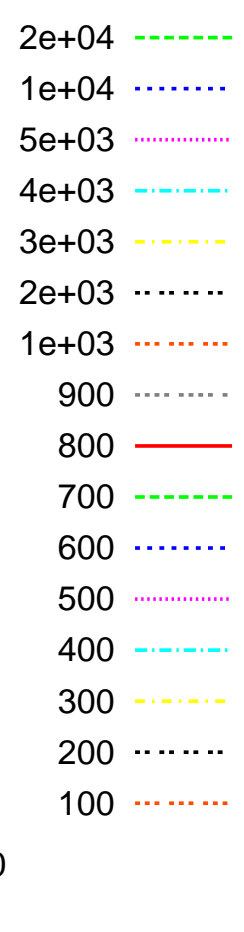

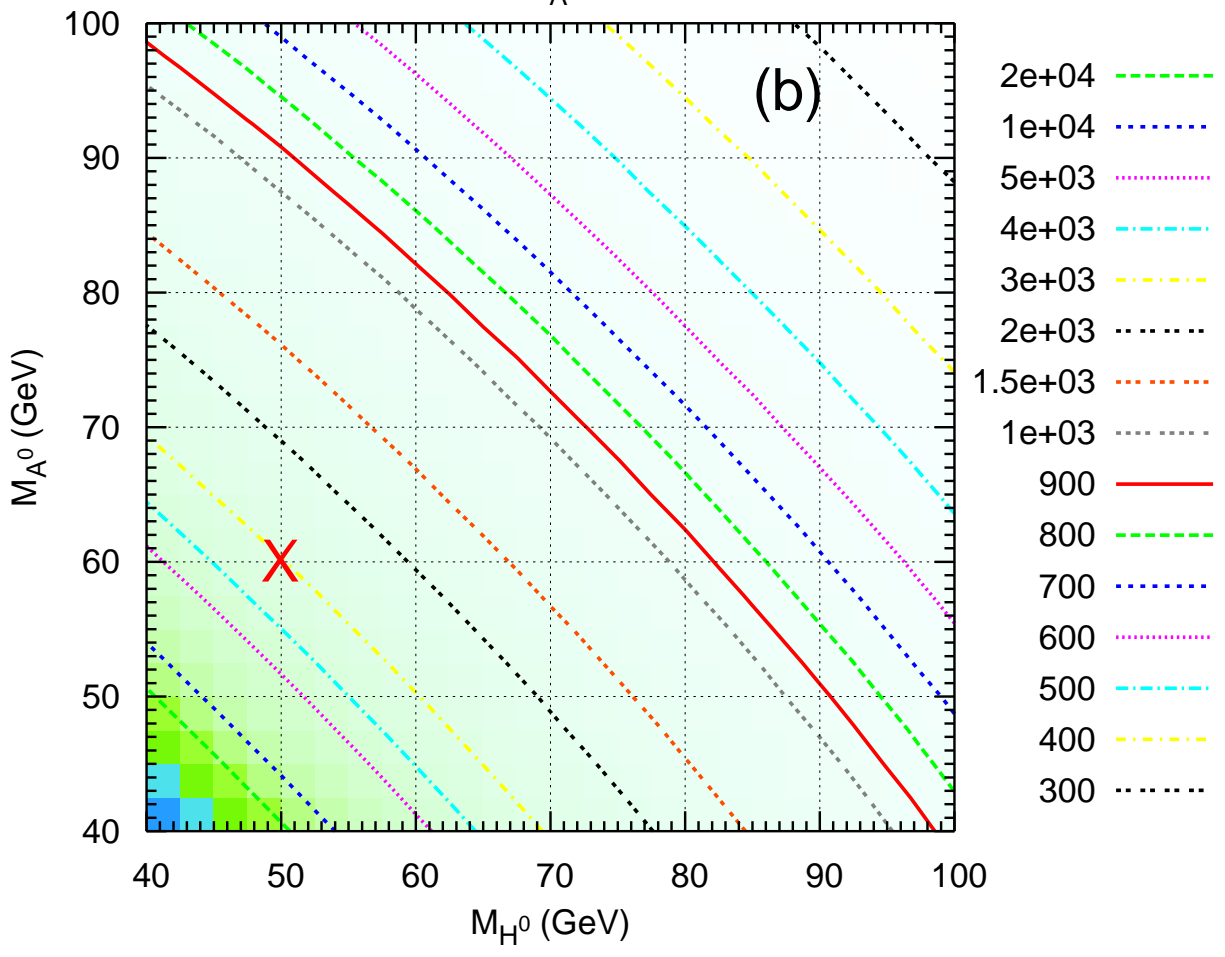

FIG. 7: Contours of the cross sections (in $f b$ units) of $A^{0} H^{+}$production (a) and of $A^{0} H^{0}$ production (b) at the LHC in the plane of the scalars' masses. The red symbol "X" denotes our benchmark point used in the collider study. Note that Fig. (a) also holds for $H^{0} H^{ \pm}$production. 


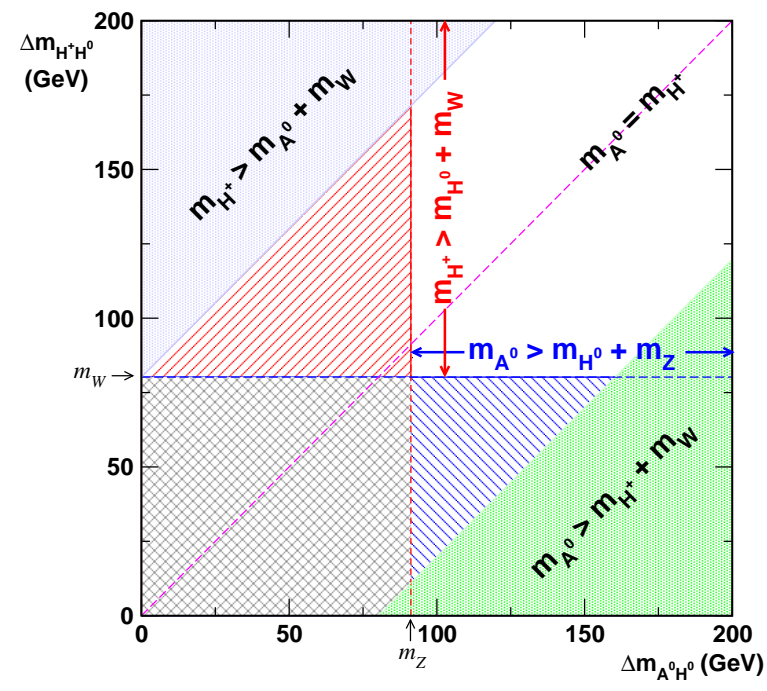

FIG. 8: Mass pattern that determines the decay pattern of $A^{0}$ and $H^{+}$.

\section{B. Decay pattern of $A^{0}$ and $H^{ \pm}$}

The decay pattern of $A^{0}$ and $H^{ \pm}$is completely determined by three mass spacings $\Delta m_{A^{0} H^{0}}, \Delta m_{H^{+} H^{0}}$ and $\Delta m_{H^{+} A^{0}}$, as shown in Fig. 8, where $\Delta m_{H^{+} H^{0}} \equiv m_{H^{+}}-m_{H^{0}}$ and $\Delta m_{H^{+} A^{0}} \equiv m_{H^{+}}-m_{A^{0}}$. Using the constraint from precision electroweak data, it has been

shown by Ref. [5] that a relationship exists between the first and second spacing in the $Z_{2}$-odd scalar spectrum

$$
\left(m_{H^{+}}-m_{A^{0}}\right)\left(m_{H^{+}}-m_{H^{0}}\right)=M^{2}, \quad M=120_{-30}^{+20} \mathrm{GeV},
$$

which implies that the charged Higgs boson should be heavier than both $A^{0}$ and $H^{0}$ so that $A^{0}$ can only decay into the $H^{0} Z^{*}$ mode. This is consistent with our choice. It corresponds to the top-left corner in Fig. 8. Of course, the precision electroweak constraint also requires the SM Higgs to be very heavy in this case, say $m_{h}>400 \mathrm{GeV}$, but that does not affect the production and decay of the dark scalar doublet particles which are independent of $m_{h}$. [Our view is that the masses of all the scalars being considered here are not necessarily constrained by precision electroweak data or dark matter relic abundance because there may be other contributions. We simply explore a set of reasonable possibilities.]

Furthermore, in order to avoid the huge QCD (quantum chromodynamics) background at any hadron collider, one has to use the leptonic decay mode to tag the signal event. Thus, in Fig. 9 we plot the decay branching ratio (BR) of $A^{0} \rightarrow H^{0} \ell^{+} \ell^{-}$and $H^{+} \rightarrow H^{0} \ell^{+} \nu$ as a function of $\Delta m_{A^{0} H^{0}}$ and $\Delta m_{H^{+} H^{0}}$ respectively. In these figures we have summed over 

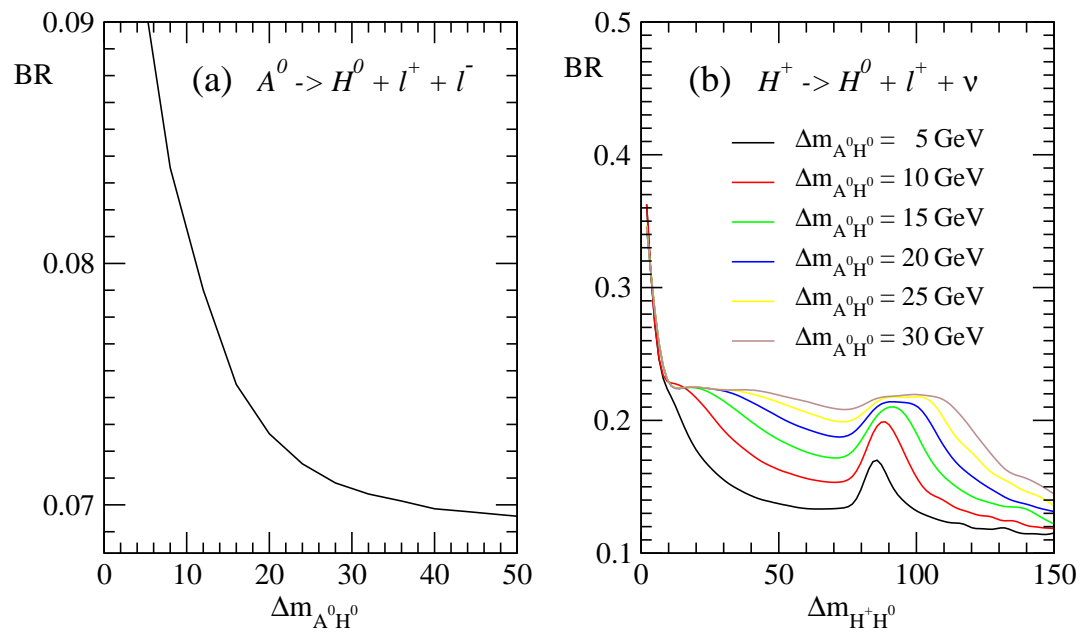

FIG. 9: Decay branching ratio of the $A^{0} \rightarrow H^{0} \ell^{+} \ell^{-}$and the $H^{+} \rightarrow H^{0} \ell^{+} \nu$ where we have summed over two lepton flavors (electron and muon). Note that $\Delta m_{H^{+} H^{0}}$ and $\Delta m_{A^{0} H^{0}}$ should satisfy the electroweak precision test bound given in Eq. 23.

two lepton flavors (electron and muon). We note that $B R\left(A^{0} \rightarrow H^{0} \ell^{+} \ell^{-}\right)$is only sensitive to $\Delta m_{A^{0} H^{0}}$. If the mass spacing is small, say $\Delta m_{A^{0} H^{0}} \sim 10 \mathrm{GeV}$, the BR is about 0.09 . As the mass spacing increases, the BR decreases because the massive quark modes, e.g. $s \bar{s}(c \bar{c}, b \bar{b})$, increase. For a very large mass spacing, the BR approaches to the BR of an on-shell $Z$-boson. The decay of $H^{+}$is a little complicated because it can decay into two modes, $H^{0} W^{(*)}$ or $A^{0} W^{(*)}$. For simplicity, we only focus on the decay mode $H^{+} \rightarrow H^{0} W^{(*)}$ in this study because the $H^{+} \rightarrow A^{0} W^{(*)}$ mode involves several particles in the final state which render the analysis of the collider signals much more complicated. Again, we note that the $\mathrm{BR}$ of $H^{+} \rightarrow H^{0} \ell^{+} \nu$ decreases as the mass spacing $\Delta m_{H^{+} H^{0}}$ increases because of the massive quark modes. The peaks in the figure are due to the mass threshold of the $W$-boson. Eventually, the BR approaches the SM $W$-boson decay branching ratio, $\sim 1 / 9$, at the large mass spacing regime.

In the following sections, we present the results of a detailed Monte Carlo simulation and determine the discovery potential of the $Z_{2}$-odd scalars at the LHC. We find that the $A^{0} H^{+} / H^{0} H^{+} / H^{+} H^{-}$pair productions suffer from huge backgrounds such that the dark scalar signals are always overwhelmed. This difficulty persists even if we lower the mass of $H^{ \pm}$. We thus focus on the $A^{0} H^{0}$ associated production in the next section. 


\section{C. $A^{0} H^{0}$ production and signature}

The neutral scalar bosons can be produced via the following process

$$
p p \rightarrow Z \rightarrow A^{0} H^{0}, \quad A^{0} \rightarrow H^{0} Z^{(*)}, \quad Z^{(*)} \rightarrow f f,
$$

which gives rise to a collider signature as $f f+\mathbb{E}_{T}$. Thus, the dominant SM background comes from two processes:

$$
\begin{aligned}
& p p \rightarrow Z Z / Z \gamma, \text { with } Z \rightarrow \nu \bar{\nu}, \quad Z / \gamma \rightarrow \ell^{+} \ell^{-}, \\
& p p \rightarrow W^{+} W^{-}, \text {with } W^{+} \rightarrow \ell^{+} \nu, \quad W^{-} \rightarrow \ell^{-} \bar{\nu},
\end{aligned}
$$

where two charged leptons in the $W W$ background are required to have the same lepton flavors. Besides these two intrinsic (irreducible) sources, there are also other reducible sources of background such as $t \bar{t}$ pair production and $W t$ associated production. For example, if the bottom quark from top quark decay escapes the detector, they will contribute to the mssing transverse momentum and thus mimic the signal events. But one can suppress these reducible backgrounds by vetoing any additional jet activity in the central rapidiy region. Hence, we focus our attention only on the intrinsic background hereafter.

Below we choose a benchmark point, $\left[m_{H^{0}}, m_{A^{0}}, m_{H^{+}}\right]=[50,60,170] \mathrm{GeV}$, to illustrate how to discover such a signature at the LHC. In order to mimic the detector, we first require both leptons in the final state to satisfy the following basic cuts:

$$
p_{T}^{\ell} \geq 15 \mathrm{GeV}, \quad\left|\eta^{\ell}\right| \leq 3.0
$$

where $p_{T}^{\ell}\left(\eta^{\ell}\right)$ denotes the transverse momentum (rapidity) of the charged lepton $\ell$. Here we have assumed a perfect detector that can precisely measure the four-momenta of the final-state leptons. The number of signal and background events, after imposing the basic cut, is given in the second column of Table III. The number of signal events is much less than the one of background events, e.g. the ratio of the signal and background event $(S / B)$

is about $10^{-3}$ where $S$ and $B$ denotes the number of the signal and background events, respectively. Obviously, additional kinematical cuts are needed to enhance the ratio of signal to background. For that, we examine the kinematics differences between signal and backgroud below and find out the optimal kinematical cuts to entangle the signal out of the background. 

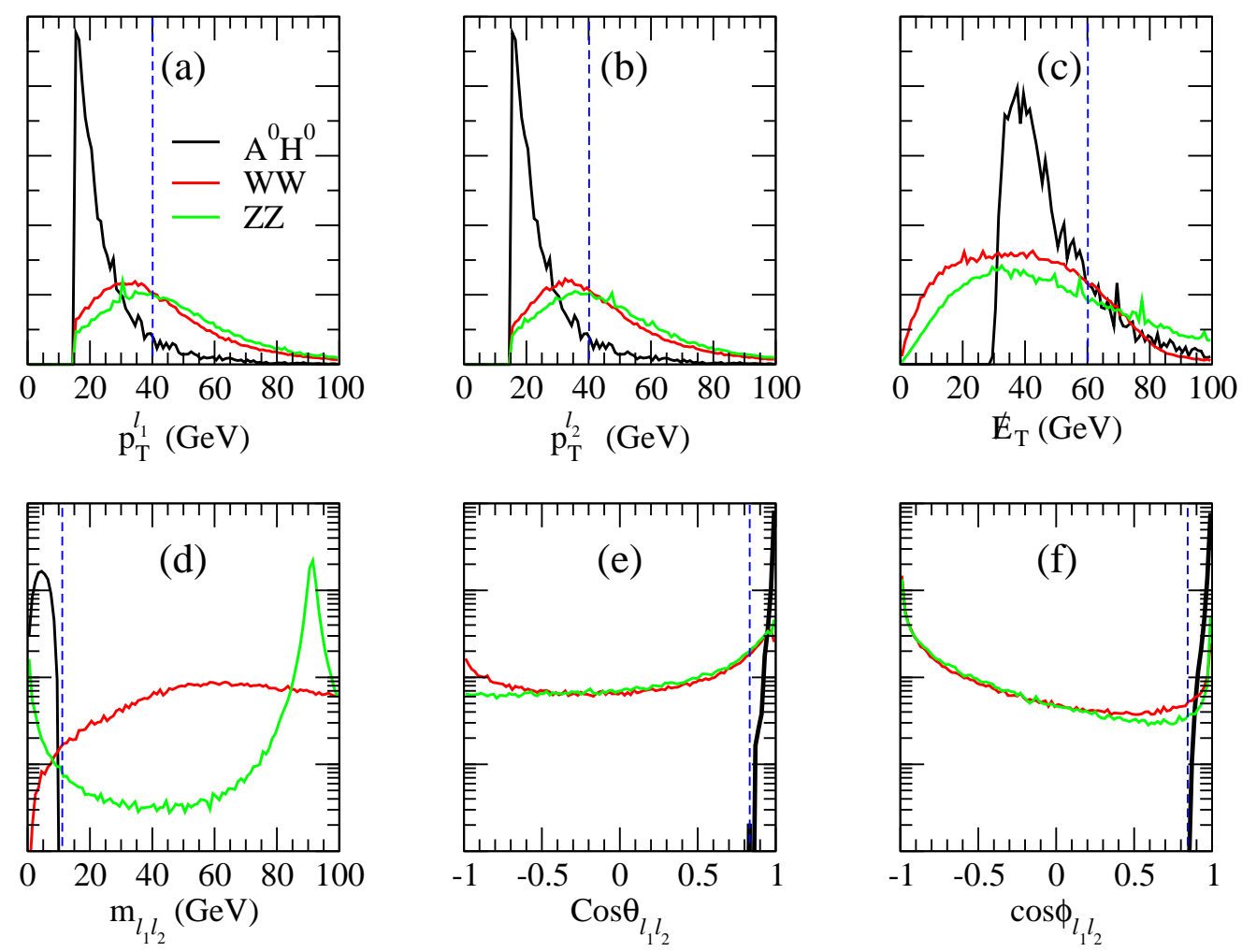

FIG. 10: (Normalized) kinematic distributions of the $A^{0} H^{0}$ production for the benchmark point $\left[m_{H^{0}}, m_{A^{0}}, m_{H^{+}}\right]=[50,60,170] \mathrm{GeV}$ (black curve), the $W W$ process (red curve) and the $Z Z$ process (green curve) after imposing the basic cuts. The blue dashed-line indicates the optimal kinematical cuts which can be used to suppress the SM background.

In Fig. 10 we present (normalized) kinematics distributions of both the signal event and the background event after imposing the basic cuts, where $\theta_{\ell \ell}\left(\phi_{\ell \ell}, m_{\ell \ell}\right)$ denotes the open angle (azimuthal open angle, invariant mass) of the two charged leptons and $\not_{T}$ the missing transverse momentum originated from the two dark scalars in the final state. We note that the kinematics of the signal events significantly differs from the one of background events in the following aspects:

- Since the two charged leptons in the signal event come from an off-shell $Z$-boson decay, they populate the small $p_{T}$ region and they prefer to move parallel to each other, see Fig. 10(a,b,e,f). On the other hand, the charged leptons of the background events have large $p_{T}$ and large open angles.

- Since the two missing scalars in the signal events are massive, one may expect a large $E_{T}$, but unfortunately, the two missing scalars prefer to move back-to-back and their 
combination actually results in a small $\mathbb{E}_{T}$ distribution, see Fig. 10(c). The distribution of the $Z Z$ background peaks around $40 \mathrm{GeV}$ while the one of the $W W$ background peaks in the large $\mathbb{E}_{T}$ region.

- The invariant mass distribution of the two charged leptons of the signal events peaks around $\sim \frac{1}{2} \Delta m_{A^{0} H^{0}}$ as they come from the decay of an off-shell $Z$-boson. The $Z Z$ background distribution exhibits two peaks: one is around $m_{Z}$ (from the on-shell $Z$-boson decay) and the other is around zero (from the virtual photon decay). The intermediate region between the two peaks is due to the interference effects between the $Z Z$ and $Z \gamma$ processes. The distribution of the $W W$ background peaks in the large invariant mass regime.

Taking advantage of the kinematic differences between the signal and the background, one can impose several optimal kinematic cuts, indicated by the blue dashed-lines in Fig. 10, to extract the signal from the background.

Based on the topological difference between the signal and background events, we impose the following optimal cuts to futher suppress the background,

$$
\begin{array}{ll}
p_{T}^{\ell} \leq 40 \mathrm{GeV}, & E_{T} \leq 60 \mathrm{GeV}, \\
\cos \theta_{\ell \ell} \geq 0.9, & \cos \phi_{\ell \ell} \geq 0.9
\end{array}
$$

At last, we require that the invariant mass of the two charged leptons satisfies the following mass window cut:

$$
0 \leq m_{\ell \ell} \leq 10 \mathrm{GeV}
$$

In Table III, we give the number of events at the LHC for an integrated luminosity of 100 $(f b)^{-1}$ of the processes metioned above as well as those of the backgound. The kinematic cuts listed in each column are applied sequentially. The two charged leptons in the final state can be either electron or muon. We take it into account by summing over the two lepton species. We also summed over three species of neutrinos in the $Z Z$ background. In order to generalize our study, we also examine the collider reach when $\Delta m_{A^{0} H^{0}}=20 \mathrm{GeV}$ and $30 \mathrm{GeV}$. As shown in the table, a staitistical significance $S / \sqrt{B} \geq 3$ can be achieved after imposing the optimal cuts and it is further increased after imposing the mass window cut for $\Delta m_{A^{0} H^{0}}=10 \mathrm{GeV}$ and $20 \mathrm{GeV}$, but slightly decreased for $\Delta m_{A^{0} H^{0}}=30 \mathrm{GeV}$ because the mass window cut, $m_{\ell \ell}<10 \mathrm{GeV}$, also cuts away $\sim 32 \%$ signal events. With an integrated 
TABLE III: Number of signal and background events, for the benchmark points $\left[m_{H^{0}}, m_{A^{0}}, m_{H^{+}}\right]=$ $[50,60(70,80), 170] \mathrm{GeV}$, at the LHC with an integrated luminosity $100 \mathrm{fb}^{-1}$. The kinematic cuts listed in each column are applied sequentially. Note that we summed over two species of charged lepton, i.e. electron and muon, for both signal and background and we also summed over three species of neutrinos in the $Z Z$ background.

\begin{tabular}{cccc}
\hline BKGD & basic & optimal & $m_{\ell \ell}<10 \mathrm{GeV}$ \\
\hline$W W$ & $1.1 \times 10^{5}$ & 110 & 62 \\
$Z Z$ & $2.1 \times 10^{4}$ & 3 & 0 \\
total & $1.3 \times 10^{5}$ & 113 & 62 \\
\hline \hline & & & \\
\hline \hline Signal & basic & optimal & $m_{\ell \ell}<10 \mathrm{GeV}$ \\
$\left(m_{H^{0}}, m_{A^{0}}\right)$ & & & 37 \\
\hline$(50,60)$ & 117 & 37 & 0.60 \\
$S / B$ & $9 \times 10^{-4}$ & 0.33 & 4.70 \\
$S / \sqrt{B}$ & 0.32 & 3.48 & 50 \\
\hline$(50,70)$ & 433 & 56 & 0.81 \\
$S / B$ & $3.3 \times 10^{-3}$ & 0.50 & 6.35 \\
$S / \sqrt{B}$ & 1.20 & 5.27 & 26 \\
\hline$(50,80)$ & 680 & 38 & 0.42 \\
$S / B$ & $5.2 \times 10^{-3}$ & 0.34 & 3.37 \\
$S / \sqrt{B}$ & 1.89 & & \\
\hline
\end{tabular}

luminosity $300 \mathrm{fb}^{-1}$, all three cases can reach $5 \sigma$ significance even before the mass window cut, therefore, LHC has a great potential to observe the dark scalar.

We note that a competition between the basic cut and mass window cut occurs. For example, the acceptance of the basic cut is large but that of the mass window cut is small for $\Delta m_{A^{0} H^{0}}=30 \mathrm{GeV}$. On the contrary, the acceptance of the basic cut is small but that of the mass window cut is large for $\Delta m_{A^{0} H^{0}}=10 \mathrm{GeV}$. When the mass gap is less than $5 \mathrm{GeV}$, the $p_{T}$ of two charged leptons is so small that most signal events fail the basic cut, and hence there is no hope to observe a signal with such a small mass gap at the LHC. 


\section{CONCLUSION}

In this work we have shown how the particles of the dark scalar doublet may be discovered at the LHC, and how their presence will affect the properties of the SM Higgs boson. From present LEP data, we obtain the bound $m_{H^{0}}+m_{A^{0}}>m_{Z}$, whereas the SM Higgs mass bound of $114.4 \mathrm{GeV}$ can be relaxed down to about $106 \mathrm{GeV}$, assuming that $m_{A^{0}}-m_{H^{0}}=10 \mathrm{GeV}$. The dark scalars dramatically affect the search of the SM Higgs boson in the intermediadte mass region, i.e. $m_{h} \sim 100-150 \mathrm{GeV}$, at the $\mathrm{LHC}$ when the decay mode $h \rightarrow H^{0} H^{0}$ opens. We find that the decay branching ratios of the usual decay modes of the SM Higgs boson, i.e. $h \rightarrow b \bar{b} / W W^{*} / Z Z^{*} / \gamma \gamma$, are highly suppressed, $\sim 60 \%$, which makes it more challenging to observe the SM Higgs boson in those usual decay modes. On the contrary, one could detect the SM Higgs boson in its decay into the dark scalars, which will escape the collider detection. We show that it is very promising to search for the SM Higgs boson through its invisible decay in the so-called weak-boson fusion process [34] which can cover most of the parameter space $\left(m_{H^{0}}, \mu_{2}\right)$ of the DSDM at the $5 \sigma$ significance. After examining the decay pattern and decay branching ratios of the dark scalars, we consider their discovery potential at the $\mathrm{LHC}$ via the process of $A^{0} H^{0}$ associated production. For $m_{H^{0}}$ of about $50 \mathrm{GeV}$ (which is also consistent with its being a dark-matter candidate), it should be observable at the LHC.

\section{Acknowledgments}

This work was supported in part by the U. S. Department of Energy under Grant No. DEFG03-94ER40837. GR thanks the Department of Physics and Astronomy, UCR for hospitality during his summer visit.

[1] N. G. Deshpande and E. Ma, Phys. Rev. D18, 2574 (1978).

[2] E. Ma, S. Pakvasa, and S. F. Tuan, Phys. Rev. D16, 1568 (1977).

[3] E. Ma, Phys. Lett. B68, 63 (1977).

[4] E. Ma, Phys. Rev. D73, 077301 (2006), hep-ph/0601225.

[5] R. Barbieri, L. J. Hall, and V. S. Rychkov, Phys. Rev. D74, 015007 (2006), hep-ph/0603188. 
[6] G. Bertone, D. Hooper, and J. Silk, Phys. Rept. 405, 279 (2005), hep-ph/0404175.

[7] E. Ma, Mod. Phys. Lett. A21, 1777 (2006), hep-ph/0605180.

[8] D. Majumdar and A. Ghosal (2006), hep-ph/0607067.

[9] L. Lopez Honorez, E. Nezri, J. F. Oliver, and M. H. G. Tytgat, JCAP 0702, 028 (2007), hep-ph/0612275.

[10] N. Sahu and U. Sarkar (2007), hep-ph/0701062.

[11] M. Gustafsson, E. Lundstrom, L. Bergstrom, and J. Edsjo (2007), astro-ph/0703512.

[12] M. Lisanti and J. G. Wacker (2007), arXiv:0704.2816 [hep-ph].

[13] E. Ma (2006), hep-ph/0607142.

[14] The ALEPH, DELPHI, L3, OPAL, SLD Collaborations, the LEP Electroweak Working Group, the SLD Electroweak and Heavy Flavour Groups, Phys. Rept. 427, 257 (2006), hepex/0509008.

[15] M. Kobel (1996), hep-ex/9611015.

[16] G. Abbiendi et al. (OPAL), Eur. Phys. J. C35, 1 (2004), hep-ex/0401026.

[17] M. Acciarri et al. (L3), Phys. Lett. B472, 420 (2000), hep-ex/9910007.

[18] Q.-H. Cao, S. Kanemura, and C. P. Yuan, Phys. Rev. D69, 075008 (2004), hep-ph/0311083.

[19] LEP Higgs Working Group (2001), hep-ex/0107032.

[20] R. Barate et al., Phys. Lett. B565, 61 (2003), hep-ex/0306033.

[21] K. Belotsky, D. Fargion, M. Khlopov, R. Konoplich, and K. Shibaev, Phys. Rev. D68, 054027 (2003), hep-ph/0210153.

[22] Q.-H. Cao, S. Gopalakrishna, and C. P. Yuan, Phys. Rev. D70, 075020 (2004), hep$\mathrm{ph} / 0405220$.

[23] G. L. Kane, T. T. Wang, B. D. Nelson, and L.-T. Wang, Phys. Rev. D71, 035006 (2005), hep-ph/0407001.

[24] S. G. Kim et al., Phys. Rev. D74, 115016 (2006), hep-ph/0609076.

[25] A. Belyaev, Q.-H. Cao, D. Nomura, K. Tobe, and C. P. Yuan (2006), hep-ph/0609079.

[26] D. Froidevaux, F. Gianotti, and E. Richter Was (1995), ATLAS Note PHYS-NO-064.

[27] F. Gianotti and I. Vichou (1996), ATLAS Note PHYS-NO-078.

[28] N. Kauer, T. Plehn, D. L. Rainwater, and D. Zeppenfeld, Phys. Lett. B503, 113 (2001), hep-ph/0012351.

[29] Q.-H. Cao and C.-R. Chen (2007), arXiv:0704.1344 [hep-ph]. 
[30] J. M. Gerard and M. Herquet, Phys. Rev. Lett. 98, 251802 (2007), hep-ph/0703051.

[31] A. Djouadi, J. Kalinowski, and M. Spira, Comput. Phys. Commun. 108, 56 (1998), hep$\mathrm{ph} / 9704448$.

[32] A. Djouadi (2005), hep-ph/0503172.

[33] A. Djouadi (2005), hep-ph/0503173.

[34] O. J. P. Eboli and D. Zeppenfeld, Phys. Lett. B495, 147 (2000), hep-ph/0009158.

[35] T. Stelzer and W. F. Long, Comput. Phys. Commun. 81, 357 (1994), hep-ph/9401258.

[36] F. Maltoni and T. Stelzer, JHEP 02, 027 (2003), hep-ph/0208156.

[37] A. Pukhov (2004), hep-ph/0412191.

[38] A. V. Semenov (2002), hep-ph/0208011. 